\title{
Contribution of ASDEX Upgrade to disruption studies for ITER
}

G. Pautasso, Y. Zhang ${ }^{(a)}$, B. Reiter, L. Giannone, O. Gruber, A. Herrmann, O. Kardaun, V.E. Lukash ${ }^{(b)}$, M. Maraschek, Y. Nakamura ${ }^{(c)}$, W. Schneider, G. Sias ${ }^{(d)}$, M. Sugihara (e) and the ASDEX Upgrade Team

Max-Planck-Institut für Plasmaphysik, EURATOM Association, D-85748 Garching, Germany

(a) ASIPP, Hefei, China; (b) Kurchatov Institute, Moscow, Russia; (c) Japan Atomic Energy Agency, Naka-shi, Ibaraki-ken, Japan; $(d)$ University of Cagliari, Italy; (e) ITER International Team, ITER, CEA-Cadarache, France

e-mail contact of main author: gabriella.pautasso@ipp.mpg.de

Abstract. The article describes the most recent contributions of ASDEX Upgrade to ITER in the field of disruption studies. (1) The ITER specifications for the halo current magnitude are based on data collected from several tokamaks and summarized in the plot of the toroidal peaking factor versus the maximum halo current fraction. Even if the maximum halo current in ASDEX Upgrade reaches $50 \%$ of the plasma current, the duration of this maximum lasts a fraction of a ms. Simulations of plasmas undergoing vertical displacement are carried out with the MHD-transport codes TSC and DINA to reproduce the measured evolution of the halo current and validate these codes for extrapolation to ITER. (2) Long-lasting asymmetries of the halo current are rare and do not give rise to large asymmetric component of the mechanical forces on the machine. Differently from JET, these asymmetries are neither locked nor exhibit a stationary harmonic structure. (3) Recent work on disruption prediction has concentrated on the search for a simple function of the most relevant plasma parameters, able to discriminate between the safe and pre-disruption phases of a discharge. For this purpose, the disruptions of the last years have been classified in groups and discriminant analysis was employed for the variable selection and the derivation of the discriminant function. (4) The attainment of the critical density for the collisional suppression of the runaway electrons seems to be technically and physically possible on our medium size tokamak. The $\mathrm{CO}_{2}$ interferometer and the AXUV diagnostic provide information on the highly $3 D$ impurity transport process during the whole plasma quench.

\section{Introduction}

Plasma disruptions represent a hazard for the structural integrity of ITER, since they will generate runaway electrons, large mechanical forces and thermal loads. The contribution of the existing tokamaks to ITER consists, firstly, in refining the characterization of these loads and their extrapolation, on the basis of physical models, and secondly, in learning to predict, avoid and mitigate disruptions. The ASDEX Upgrade (AUG) research program 
covers these specific topics and this paper reports on significant progress made recently in these areas. For work on disruption avoidance, not discussed in this contribution, the reader is referred to reference [1].

\section{Halo current}

The halo current causes large electromagnetic forces and, from an engineering point of view, its magnitude and spatial distribution must be known for dimensioning the intercepted structures. The analysis of the halo current evolution in AUG is of particular interest for the following reasons: The device is equipped with a large number of detectors which allow the reconstruction of the current profile on the divertors and on the heat shield in different toroidal sectors. AUG has always reported halo current magnitudes larger than those in other tokamaks ([2], [3]); the reasons for this are still unknown. Moreover, the halo current exhibits large toroidal asymmetries correlated with strong MHD activity. Kink modes are believed to cause large asymmetries of the halo current [4] and to drive the large horizontal forces observed up to now only in JET [5]-[6]. At present, no physics-based model is available for the prediction of the amplitude of these modes in ITER and any further understanding of their driving mechanism is valuable.

The ITER specifications for the expected magnitude of the halo current and its degree of toroidal asymmetry have been based up to now on data collected from several tokamaks and summarized in the plot of the toroidal peaking factor, TPF, versus the maximum halo current fraction, $\max \left(I_{h}\right) / I_{p, 0}$ (see figure 64 of [2] and figure 42 of [3]). This TPF is, more precisely, taken at the time of the maximum halo current fraction; in this work we define $T P F_{m} \equiv T P F\left(t_{\max \left(I_{h}\right) / I_{p, 0}}\right)$. Currently, there is no physics based specification for the time history of $I_{h}$ and TPF; nevertheless, the information on the duration of $\max \left(I_{h}\right)$ and $T P F_{m}$, that is on the impulse exerted, is required for the design of the mechanical structures, since their dynamic response depends also on the load waveform. The data points in the aforementioned plot reach the $\max \left(I_{h}\right) / I_{p, 0} \times T P F_{m}=0.75$ boundary [2], which represents a challenging load for the mechanical design of the structures, if applied during the whole current quench. A self-consistent simulation of the whole vertical displacement (VD), following or preceding a disruption, is required to predict the halo current time history and detailed code benchmark is in progress (see section 3). The value of $\max \left(I_{h}\right) / I_{p, 0}$ can reach $50 \%$ in AUG, as it is shown in figure 1 , although the mean of its statistical distribution amounts to $27 \%$ for unmitigated disruptions; mitigated disruptions have a much lower halo current. VDEs and VDs following centered disruptions have a similar distribution density on this figure. This happens because often the thermal quench of centered disruptions leaves residual thermal energy in the plasma

and the fast decay of the current starts only later when the plasma has displaced vertically and is limited by the divertor. This type of VD needs to be considered as one of the ITER disruption scenarios, since so far only the VD with fast current quench after thermal quench has been examined [7]; in this case the VD occurs at reduced plasma 
current and generates a small fraction $I_{h} / I_{p, 0}$.

Several discharges are close to the $\max \left(I_{h}\right) / I_{p, 0} \times T P F_{m}=0.75$ boundary. Independently of where the discharge is located along the boundary, the large $\max \left(I_{h}\right) / I_{p, 0}$ or/and $T P F_{m}$ last $0.2-0.4 \mathrm{~ms}$, and they typically coincide with the appearance of a large perturbation of the magnetic configuration with a toroidal mode number $n=1$. The $n=1$ halo current structure survives for a few ms, it is generally locked or rotates slowly with a frequency $\leq 1 \mathrm{kHz}$, at most 1.5 times around the torus. The rotation is mostly in the counter- $I_{p}$ direction but cases with co- $I_{p}$ are also known.

The difference between the high $\max \left(I_{h}\right) / I_{p, 0}$ and the high $T P F_{m}$ data points along the boundary seems to be due to the different amplitudes and rotation frequency of the $n=1$ halo current perturbation and its occurrence in time, with respect to $t_{\max \left(I_{h}\right) / I_{p, 0}}$. How this depends on the plasma parameters is not known. If the current decay is faster than the shrinking of the plasma cross section (large safety factor) and strong interaction with the plasma wall is missing, such as after massive gas injection, then the strong MHD event is not visible and the halo current is toroidally symmetric.

In order to find cases with long lasting asymmetries, the following asymmetry (A) factor has been defined (following [8] and [9]) and calculated for the shots of the database:

$$
A(t)=\max _{i}\left[I_{i}(t)-I_{h}(t)\right] / I_{p, 0} \equiv[T P F(t)-1] I_{h}(t) / I_{p, 0}
$$

The 10 cases with the largest $\int A(t) d t$ have been analyzed in more detail: They comprise an equal number of VDEs and VDs after centered disruptions, ohmic and auxiliary heated plasmas (with up to $7.5 \mathrm{MW}$ ), with different values of $q_{95}$. The analysis of the fast Mirnov coil measurements show that the origin of the large halo current asymmetry can vary: In addition to the cases in which a large amplitude but short lived $n=1$ mode grows, other scenarios are found. In some discharges, low $m-$ and $n$-number modes, rotating up to the thermal quench, seem to survive it, slow down considerably and cause the asymmetry of the halo current. This case is rather common for VDEs with NBI input torque and it is illustrated in figure 2 (a): An $m / n=2 / 1$ mode is rotating during the VDE, it slows down considerably when the vertical position of the plasma current center $z_{\text {curr }} \sim 0.3 \mathrm{~m}(\mathrm{t}=2.2785 \mathrm{~s})$ and looses its regular sinusoidal form. Between $\mathrm{t}=2.280 \mathrm{~s}$ and the end of the current quench the toroidal mode number show both even and odd components; the poloidal mode number cannot be determined after $2.280 \mathrm{~s}$ because the coil measurements are saturated in this case. Figures 2 (b) and (c) show that the halo current modulation (local measurement and toroidal average respectively) is correlated with the $B_{\theta}$ oscillations and that the amplitude of the poloidally localized toroidal asymmetric component can be much larger than the symmetric one.

In none of the discharges analyzed a stationary mode with a well defined $m / n$ structure develops, contrary to the JET VDEs, in which several periods of a sinusoidal modulation of the halo current is observed [10]. Moreover, large horizontal forces on the AUG vessel have never been recorded up to now. 


\section{Simulation of VDEs}

Simulations of AUG VDEs and centered disruptions are currently being carried out [11][13] with the MHD-transport codes DINA [14] and TSC [15]. These codes rely on 2D selfconsistent models (from the electromagnetic point of view) of the plasma and surrounding conductors and can predict the evolution of the halo current if the halo region properties, namely width and temperature are given. For existing experiments, the halo properties can be chosen to fit the experimental measurements; a rational choice of these parameters can then be used for ITER simulations.

This work is part of the effort made by the existing tokamaks in the framework of the ITPA MHD Topical Group to test the capability of the respective halo models to reproduce the spatial and temporal evolution of the measured halo current, and to characterize the development of the halo region. This benchmark will contribute to legitimize the use of these codes for the prediction of ITER halo currents.

\section{Prediction of disruptions}

The plasma control system on ITER foresees a subsystem for exception handling [16], which should be able to perform a controlled emergency shut-down or trigger a fast mitigated termination. Every tokamak implements already protection measurements and has some kind of disruption alarm; however no reliable disruption prediction system exists yet in present-day machines and there is no general consensus on a concept.

Strictly speaking, a major disruption is not the result of a deterministic process. Several cases of AUG discharges repeated with same machine and - as far as controllable - plasma parameters are known, which can undergo either a minor or a major disruption at a certain point in the discharge. Figure 4 shows an example of two equal discharges disrupted, one at high $\beta$ and the other one at low $\beta$ after a VDE induced by a minor disruption. Predicting major disruptions is therefore a statistical problem. The onset of the instability, major or minor, seems to be more reproducible.

Several publications on disruption prediction based on statistical methods have appeared in the last decade (see for example [17]-[23] publisher after 2005). They have shown that with a limited number of machine and plasma parameters, available in real time, and a database of safe and disruptive discharge phases, it is possible to build a complex function which can predict the disruption occurrence with a relatively small rate of false alarms (some \%) and a large success rate (80-90\%). For example, a neural network trained with the discharges of the last years has been continuously updated, supplemented with a novelty detection algorithm and is available for the AUG operation [24].

Although a physics-based algorithm, which does not have to rely on a large database of disruptions, would be desirable, as a matter of fact, there is not a unique disruption

precursor, which can be used as disruption alarm. Recent work in this area, on AUG, has concentrated on the search for a simple function of the most relevant plasma parameters, 
which can discriminate between the safe and pre-disruption phases of a discharge [25]. The aim of this work is to test if the boundary can be expressed by a power law scaling and if this reflects the known physics.

The disruptions of the last four years have been analyzed and classified according to their physical causes. Most of the disruptions in this period are of the edge-cooling type or due to loss of vertical control ( $52 \%$ and $36 \%$ respectively); disruptions following the beta limit $(3 \%)$ and impurity accumulation ( $8 \%)$ form two smaller groups. The plasma state is described by the following variables: internal inductance $\left(l_{i}\right)$, line-averaged electron density normalized by the Greenwald limit $\left(n_{e} / n_{G W}\right)$, loop voltage $\left(U_{\text {loop }}\right)$, radiated power normalized by the total input power $\left(P_{\text {rad }} / P_{i n}\right)$, the $\mathrm{H}$ factor, beta normalized $\left(\beta_{N}\right)$, plasma safety factor $\left(q_{95}\right)$, elongation, triangularity and plasma form (limiter/divertor).

The VDEs can be predicted by monitoring the vertical plasma position, since they are relatively slow in the shot range analyzed, with respect to the control system cycle time. Discriminant analysis is applied to each of the other three disruption groups and to the safe plasma samples. Performing a pre-classification is quite important in this case, since an effective discrimination is only possible with this method when the two groups can be separated by a (log-)quadratic or (log-)linear function of the significant variables.

Each group is assumed to have a multivariate normal distribution

$$
f_{g}(\mathbf{x})=(2 \pi)^{-\mathrm{p} / 2}\left|\boldsymbol{\Sigma}_{\mathrm{g}}\right|^{-1 / 2} \exp \left[-0.5 \mathrm{~d}_{\mathrm{g}}^{2}(\mathbf{x})\right]
$$

where $d_{g}^{2}(\mathbf{x})=\left(\mathbf{x}-\mathbf{X}_{\mathrm{g}}\right)^{\prime} \boldsymbol{\Sigma}_{\mathrm{g}}^{-1}\left(\mathbf{x}-\mathbf{X}_{\mathrm{g}}\right)$ is the distance of the observation $\mathbf{x}$ to the mean of group $g, \mathbf{X}_{\mathrm{g}}$, and $\boldsymbol{\Sigma}_{\mathrm{g}}$ is the within-group covariance matrice. From a geometrical point of view, the intersection between the two $f_{g}$ define a multi-D surface (quadratic function), which reduces to a hyper-plane (linear function) if the covariance matrices of the two distributions are equal.

For investigation purposes, the two distributions can be forced to be equal by assuming a common covariance matrix and estimating it as the linear combination of the $\boldsymbol{\Sigma}_{\mathrm{g}} \mathrm{s}$, that is $\boldsymbol{\Sigma} \equiv\left[\left(\mathrm{n}_{1}-1\right) \boldsymbol{\Sigma}_{1}+\left(\mathrm{n}_{2}-1\right) \boldsymbol{\Sigma}_{2}\right] /\left(\mathrm{n}_{1}+\mathrm{n}_{2}-2\right)$ where $n_{1}$ and $n_{2}$ are the number of samples in each of the two groups. This simplifying assumption allows the derivation of the linear discriminant function describing the boundary between the two groups. Moreover the statistical significance of the variables can be determined by means of the Wilks' Lambda method [27] implemented in SAS [28].

The most interesting results of this work are that: (1) few plasma parameters $\left(l_{i}\right.$, $n_{e} / n_{G W}, U_{\text {loop }}$ or the $\mathrm{H}$ factor in order of importance) are necessary to predict the egdecooling disruptions, while the addition of other variables does not significantly decrease the prediction error rate (see figure 5); (2) linear and quadratic functions of a few (3-5) plasma parameters can discriminate between safe and pre-disruption samples with a small error rate and predict the edge-cooling disruptions (see figure 6). The variables, which allow to discriminate between safe and pre-disruption states of one class of disruptions should be universal among tokamaks. The coefficients in the formula describing the boundary might need to be fine-tuned for each device. 
Discriminant analysis, with the same variables as above, is not successful in predicting disruptions following beta limit and impurity accumulation. Nevertheless, beta limit disruptions are always preceded by the development of detectable magnetic modes, which must be used for their prediction. Impurity accumulation does not necessarily or rapidly lead to disruption, and it can easily be detected by the ratio between the radiated power from the plasma center and plasma periphery. The AUG control system has then the possibility of turning on central heating or starting the premature shut-down of these plasmas [29].

\section{Experiments of massive gas injection}

The critical electron density, $n_{c}$, required to assure the collisional suppression of runaway electrons in ITER disruptions is between one and two orders of magnitude larger than the one needed for forces and heat load mitigation. Progress has been made in AUG in attaining an effective electron density closer to the critical one, namely $n_{e, \text { eff }} / n_{c} \sim 24 \%$, by injecting 3.3 bar.l of neon [31]. At this large amount of injected impurities, the fuelling efficiency remains at the level of $20 \%$ for plasmas with a modest thermal energy $(<0.4 \mathrm{MJ})$. Nevertheless, a significant decrease of the fuelling efficiency with increasing plasma thermal energy has been observed at large amounts of injected helium and neon atoms. This could be due to the $\nabla B$ drift, which has been observed to favor deeper fuel penetration and higher fuelling efficiency of pellets when launched from the high field site. Presently, the attainment of $n_{c}$ in the medium size tokamak AUG seems to be technically and physically possible and other fast valves are planned to be installed on the high and low field side.

It is unrealistic to assume that the plasma density could be increased up to $n_{c}$ over the whole plasma cross section before the thermal quench, since it has been observed in several devices [32]-[34] that the thermal quench is triggered when the impurity bulk has reached a flux surface with $q \sim 2$. Nevertheless, this does not exclude a-priori that MGI can be used for runaway suppression, providing that the core density increases after the thermal quench fast enough to stop the runaway electrons and their generation in the plasma center. Measurements indicate that during the thermal quench the mobility of the impurities increases radially and toroidally, and that during the current quench strong radiation is localized at or close to the plasma core. Nevertheless, no code can presently simulate the complex MHD and transport phenomena triggered by MGI and predict the redistribution of the impurity ions in a plasma.

The $\mathrm{CO}_{2}$ interferometer and the AXUV cameras have been valuable diagnostics in the study of impurity assimilation and redistribution.

The $\mathrm{CO}_{2}$ interferometer is located in sector $11(\Delta \phi=\pi / 4$ away from the valve) and provides density measurements along three vertical cords, whose geometry is shown in

the inset of figure 7 . For moderate amounts of injected neon $\left(N_{i n j} \leq 4 \times 10^{22}\right)$, the line averaged electron density measured by the V-1 and V-2 channels evolve similarly 
during the entire disruption, indicating a relatively fast poloidal redistribution of the density. Helium has the tendency to build a MARFE-like structure on the high filed side, particularly at low-moderate injected amounts, as shown in figure 8 . In this case, the density rise (phase I) is completed when large MHD activity (phase II) starts. The edge chord V-3 shows a strongly modulated but gradual decay of the density, apparently not accelerated by the MHD activity. The chord V-2 views a region of high density, also seen by the fast CCD camera as a radiating structure poloidally localized on the inner wall (MARFE). This strong poloidal asymmetry persists during phase II.

At large amounts of injected impurities, independent of the thermal energy and type of gas, the density distribution is poloidally and toroidally very asymmetric, as shown in figure 7 , implying that multiple valves are needed to distribute the density further. The fast current decay and the low mobility of the impurities constitutes physics limits to their redistribution in the AUG plasma. Care was taken in calculating the fuelling efficiency by time averaging the $\mathrm{V}-1$ and $\mathrm{V}-2$ measurements in the time interval $\Delta t_{F_{e f f}}$ (figure 7), i.e. between the thermal quench onset and the time at which the plasma current has decayed to $20 \%$ of its predisruptive value [31].

As illustrated in figure 11, the AXUV diagnostic consists of several diode arrays viewing the plasma in sector 13 , where the valve is situated $(\phi=0)$ and in second 5 , located a toroidal angle $\Delta \phi=\pi$ apart. The AXUV measurements (figure 11) clearly show the highly 3D nature of impurity transport. During the few ms of the cooling phase (defined as the time interval between the arrival of the impurities at the edge and the onset of the thermal quench), a highly radiating structure, first localized at the plasma edge and slowly moving inwards, is made visible by the vertical AXUV camera at $\phi=0$. This structure can be identified with the steep positive gradient of the impurity atoms diffusing into the plasma and with the negative gradient of the plasma temperature decaying to a few eV towards the plasma edge because of the large radiation. The radial movement of the cold front can be understood in terms of radial diffusion of the injected impurities. Simulations carried out with the SOLPS code [35] yield an evolution of the density and temperature time history (figure 9) comparable with the experimentally observed one and visualize the radial impurity penetration. This emissivity front moves towards the plasma center, does not penetrate up to it but reaches a surface with $q \sim 2$ within 2 ms; afterward the thermal quench sets in. Filamentary structures detach from this front, they are short lived and cannot be identified in the sector $\Phi=\pi$.

During the cooling phase the plasma radiates between 10 and $70 \%$ of its thermal energy mostly close to the valve location. The attempt of explaining this large variability range in terms of different plasma or gas parameters has not been successful up to now. Figure 10 shows that neither $q_{95}$ nor $N_{i n j}$ or the gas sort can explain the different magnitude of plasma energy loss during the cooling quench.

The toroidal distribution of the impurities is not known precisely. Nevertheless it is observed that the power radiated in the sector $\Phi=\pi$ during this phase is a very small fraction of the power radiated in the sector of gas injection. This must be a consequence, due to their low temperature, of the low toroidal ion mobility. 
During the thermal quench (lasting $1 \mathrm{~ms}$ ) the impurity particles seem to diffuse around the torus and penetrate into the plasma: The thermal energy, still confined in the plasma, heats up the impurity ions and neutrals at the plasma edge, and transiently increases their temperature and mobility. As a matter of fact, both vertical AXUV cameras $\Delta \phi=\pi$ apart record a radiated emission one order of magnitude larger than during the rest of the disruption. The tomographic reconstruction of the radiation profile in sector 5 during the thermal and beginning of the current quenches show a rapid poloidal and radial redistribution of the impurity atoms; during the current quench after helium injection and to a less degree after neon puff, the radiated power comes from the plasma bulk.

\section{Summary}

In the area of disruption studies for ITER, the main AUG achievements of the last two years can be summarized as follows:

(1) The halo current can reach $50 \%$ of the plasma current, independently of the disruption type, but this maximum value lasts a fraction of a ms. The question whether 2D MHD transport codes like DINA and TSC can reproduce $\max \left(I_{h}\right) / I_{p, 0}$ is under investigation.

(2) The toroidal halo current asymmety is typically caused by a large magnetic perturbation with a toroidal mode number $n=1$, appearing at the maximum of the vertical displacement during the current quech. This perturbation lasts one millisend. Long-lives asymmetries are an exception in AUG and they neither exhibit a regular periodic structure nor are locked. Large horizontal forces on the AUG vessel have never been recorded up to now.

(3) A preliminary classification of disruptions in groups, which reflects their physical cause, allows to apply the discriminant analysis to find functions describing the boundary between each group and the safe plasma states. Disruptions caused by edge-cooling can be predicted by a simple linear or quadratic function of 3-5 plasma variables. Disruptions following beta limit and impurity accumulation are more unpredictable and additional precursors (MHD, for example) must be used for their forecast.

(4) The attainment of the critical density in the medium size tokamak AUG seems to be technically and physically possible and other fast valves are planned to be installed on the high and low field side. They will favor the redistribution of the injected impurities around the torus, which is otherwise prevented by the low ion temperature and mobility.

\section{References}

[1] B. Esposito et al., contribution EXW/10-2Ra, this conference

[2] ITER Physics Expert Group on Disruptions, Plasma Control, MHD and ITER Physics Basis Editors, Nucl. Fusion 39 (1999) 225 
[3] T.C. Hender et al., Nuclear Fusion 47 (2007) 128-202

[4] N. Pomphrey et al., Nuclear Fusion 38 (1998) 449-466

[5] L.E. Zakharov, Physics of Plasma 15 (2008) 062507

[6] P. Noll et al., in Proceedings of the 19th Symposium on Fusion Technology, Lisbon, edited by C. Varandas and F. Serra (Elsevier, Amsterdam, 1996), Vol. 1, p. 751

[7] G. Sannazzaro et al., Structural load specification for ITER tokamak components, Symposium on Fusion Engineering, 23rd IEEE/NPSS (1-5 June 2009) San Diego, USA

[8] S.N. Gerasimov et al., contribution P4.121, 37th EPS Conference on Plasma Physics and Controlled Fusion (21-25 Jun 2010), Dublin, Ireland

[9] T. Hender's private communication

[10] V. Riccardo et al., contribution I5.118, 37th EPS Conference on Plasma Physics and Controlled Fusion (21-25 Jun 2010), Dublin, Ireland, to be published in PPCF

[11] V.E. Lukash et al., contribution P4.140, 37th EPS Conference on Plasma Physics and Controlled Fusion (21-25 Jun 2010), Dublin, Ireland

[12] Y. Nakamura et al., contribution P2.184, 37th EPS Conference on Plasma Physics and Controlled Fusion (21-25 Jun 2010), Dublin, Ireland

[13] L. Bandyopadhyay et al., contribution ITR/P1-16, this conference

[14] R.R. Khayrutdinov and V.E. Lukash, J. Comput. Physics 109 (1993) 193-201

[15] S.C. Jardin et al., J. Comp. Physics 66 (1986) 481

[16] J.A. Snipes et al., Fusion Engineering and Design 85 (July 2010) 461-465

[17] B. Cannas et al., Nuclear Fusion 46 (2006) 699

[18] B. Cannas et al., Nuclear Fusion 47 (2007) 1559

[19] Wang Hao et al., Chinese Physics, 16 (12) (2007) 3738-04

[20] A. Murari et al., Nuclear Fusion 49 (2009) 055028

[21] G.A. Ratta' et al., Nuclear Fusion 50 (2010) 025005

[22] B. Cannas et al., Nuclear Fusion 50 (2010) 075004

[23] A. Murari et al., Nuclear Fusion 50 (2010) 05500

[24] B. Cannas et al., 27th SOFT (27 Sep - 1 Oct 2010), Porto, Portugal

[25] Y. Zhang et al., contribution P2.194, 37th EPS Conference on Plasma Physics and Controlled Fusion (21-25 Jun 2010), Dublin, Ireland

[26] C.M. Bishop, Neural Networks for Pattern Recognition, (1995) Clarendon Press, Oxford

[27] A.M. Kshirsagar, Multivariate analysis, (1972) Marcel Dekker Inc., New York and Basel

[28] SAS Institute Inc., Cary, NC, USA. SAS/STAT 9.2 User's Guide, Second Edition, 2009, procedures REG and DISCRIM

[29] A. Kallenbach et al., Nuclear Fusion 49 (2009) 045007

[30] T.C. Hender et al., Nuclear Fusion 47 (2007) 128-202

[31] G. Pautasso et al, Plasma Physics and Control Fusion 51 (2009) 124056 
[32] E. Hollman et al., Phys. Plasmas 14 (2007) 012502

[33] C. Reux et al., Nuclear Fusion 50 (2010) 095006

[34] B. Reiter, PhD Thesis presented at the Faculty of Physics, Ludwig-MaximiliansUniversität, Munich, 21.5.2010

[35] G. Pautasso et al., contribution EX/P9-1, Proc. of the 22nd IAEA Conference Fusion Energy, Geneva, Switzerland, (October 2008 (IAEA, Vienna), Vol. IAEA-CN-165 (2008) 


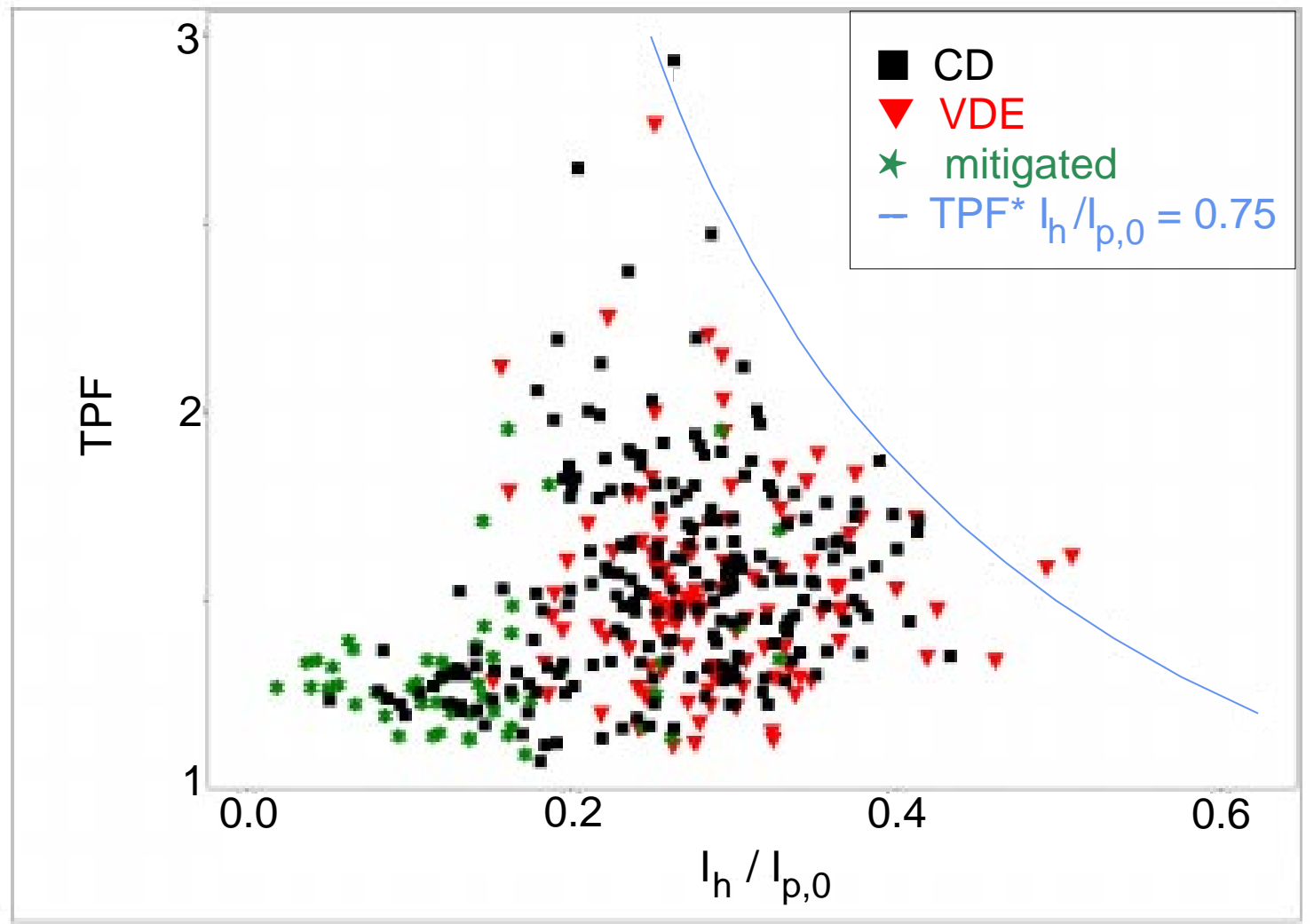

Figure 1: Toroidal peaking factor $\left(T P F_{m}\right)$ versus maximum halo current fraction $\left(\max \left(I_{h}\right) / I_{p, 0}\right)$ for different types of disruptions. 

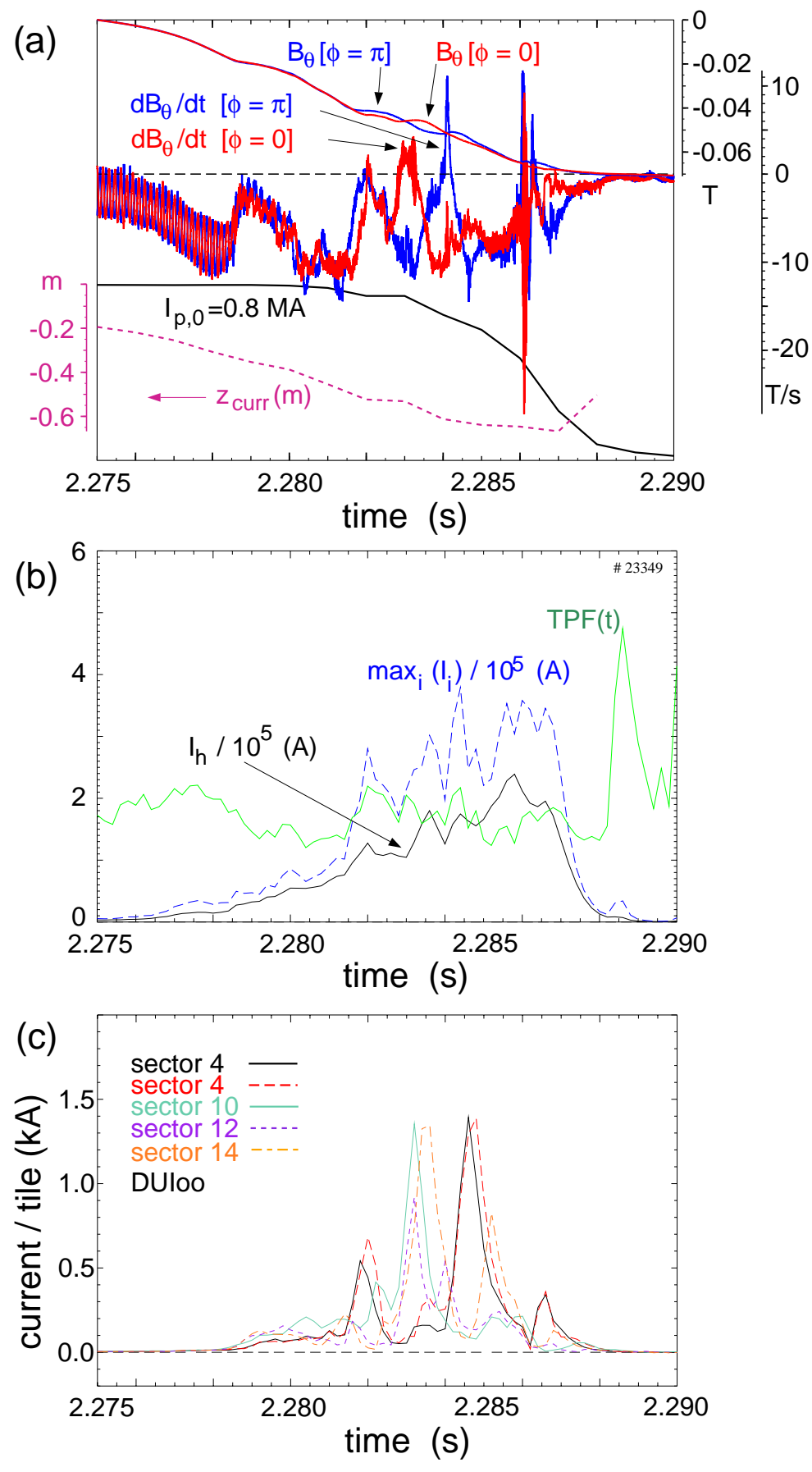

Figure 2: Time traces of (a) plasma current $\left(I_{p}\right)$, vertical position of the current center $\left(z_{\text {curr }}\right)$, measurements from two Mirnov coils $\left(d B_{\theta} / d t\right)$ located at the same poloidal position $(\theta=0)$ in two sectors $\Delta \phi=\pi$ apart, and their time integral; (b) the toroidal peaking factor $(T P F)$, the toroidally averaged halo current $\left(I_{h}\right)$, the maximum halo current measured in a sector extrapolated to the 16 sectors $\left(\max _{i}\left(I_{i}\right)\right)$, and $(\mathrm{c})$ the current per tile measured by the DUIoo shunt array (upper part of inner divertor plate) for a discharge with large $\int A(t) d t$. 

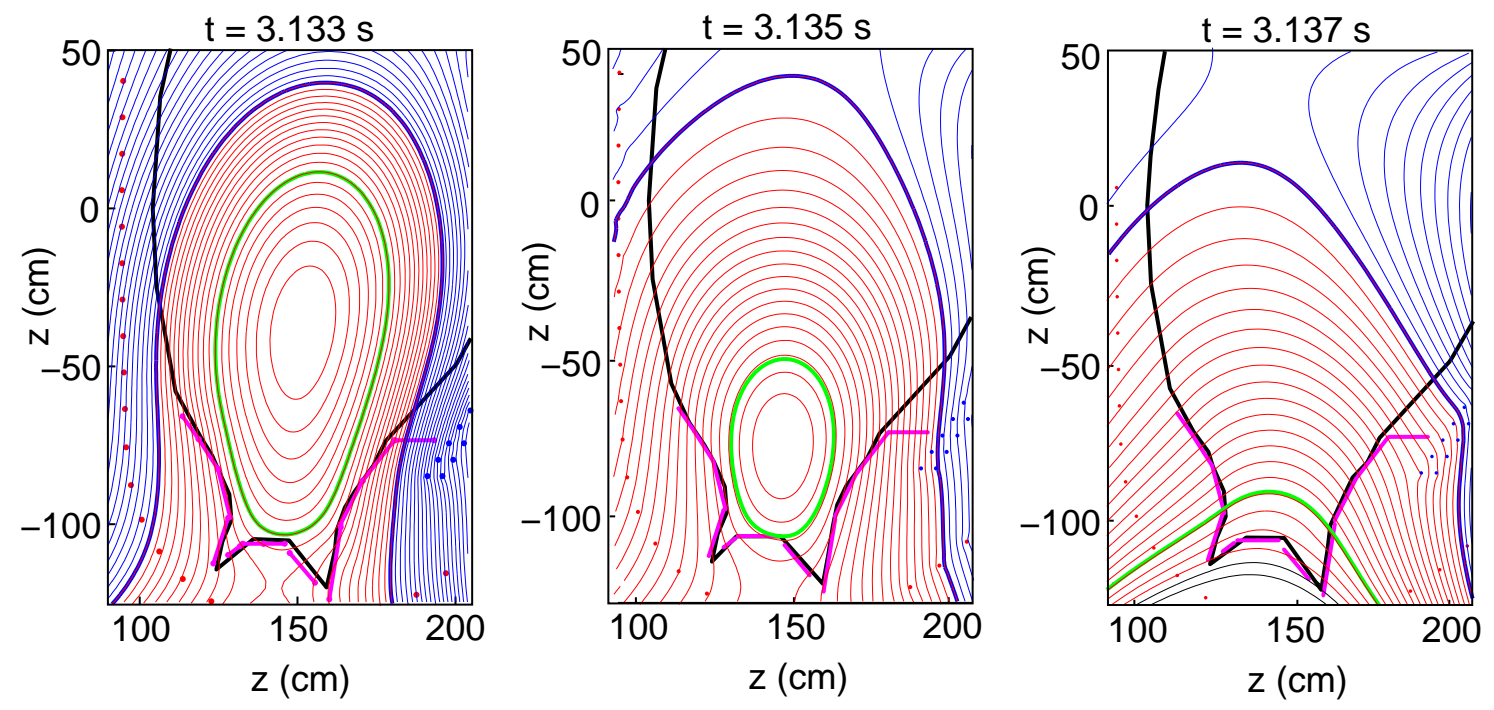

Figure 3: Evolution of the plasma equilibrium during a VDE (\# 25000) as reconstructed by the DINA code [11]. 

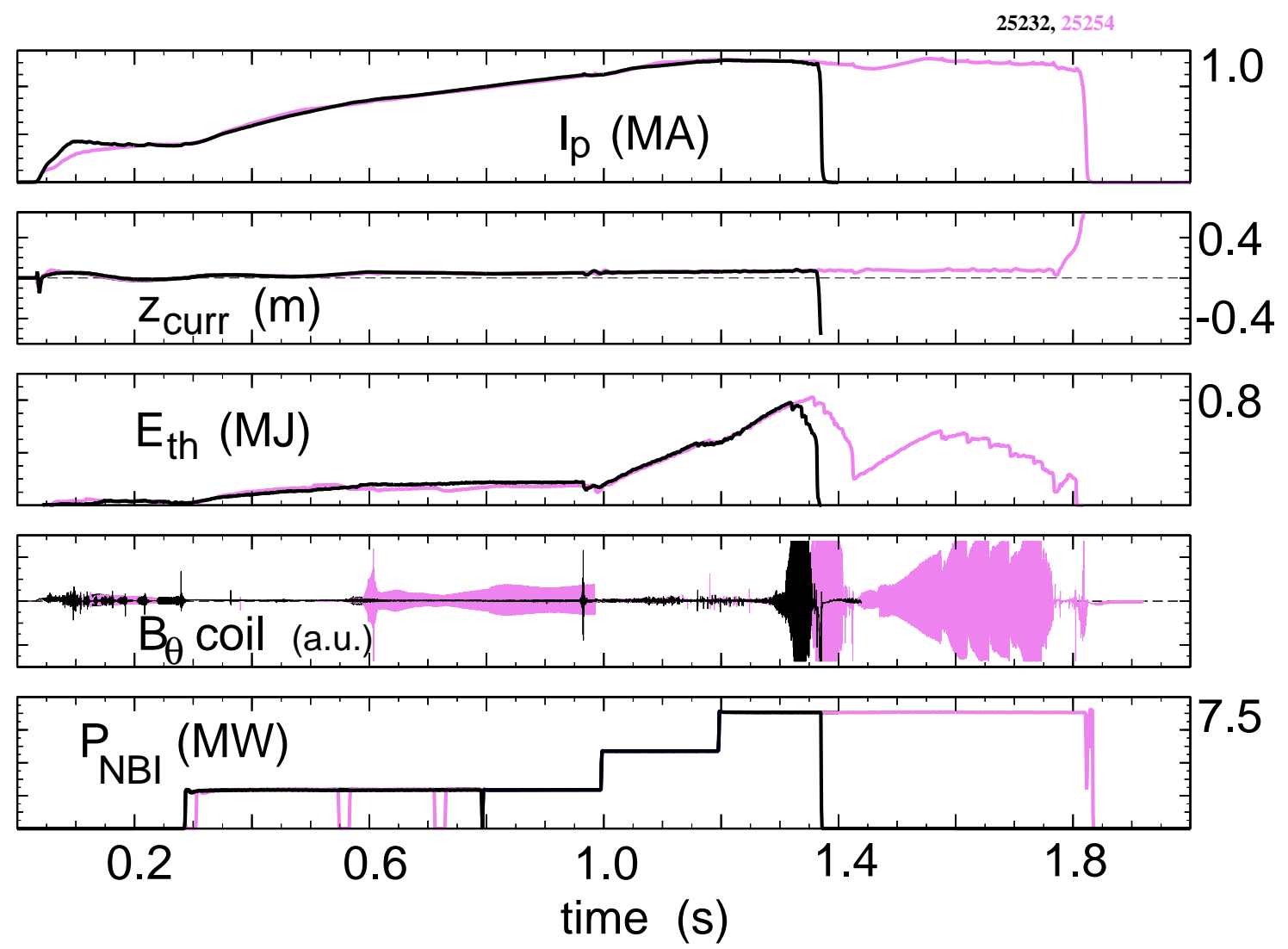

Figure 4: Discharges repeated with the same controllable plasma and machine parameters and disrupted, one at large thermal energy, $E_{t h}$, (shot 25232) and the other one at low $E_{t h}($ shot 25254) after a minor disruption induced VDE. 


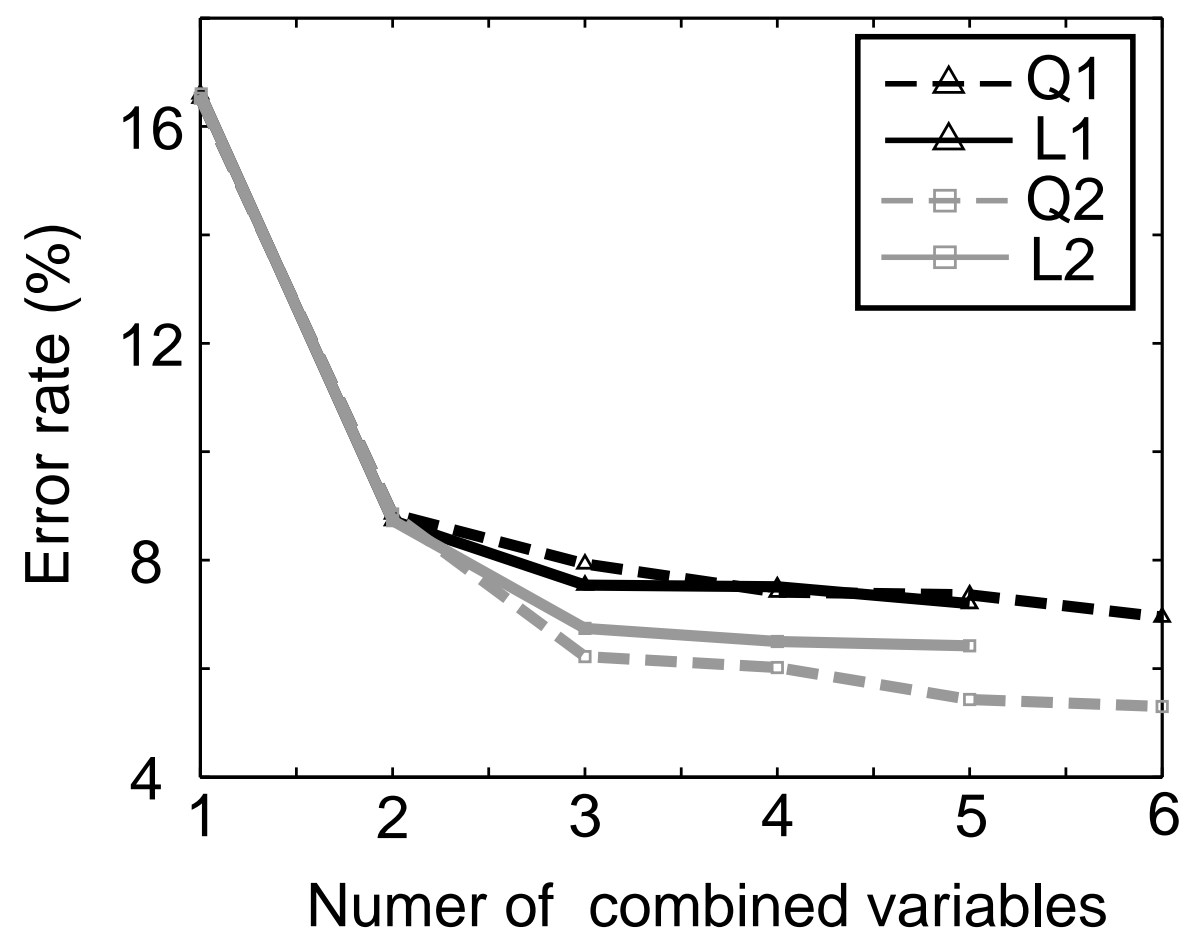

Figure 5: Misclassification error rate of two linear (L1, L2) and two quadratic functions (Q1, Q2). 


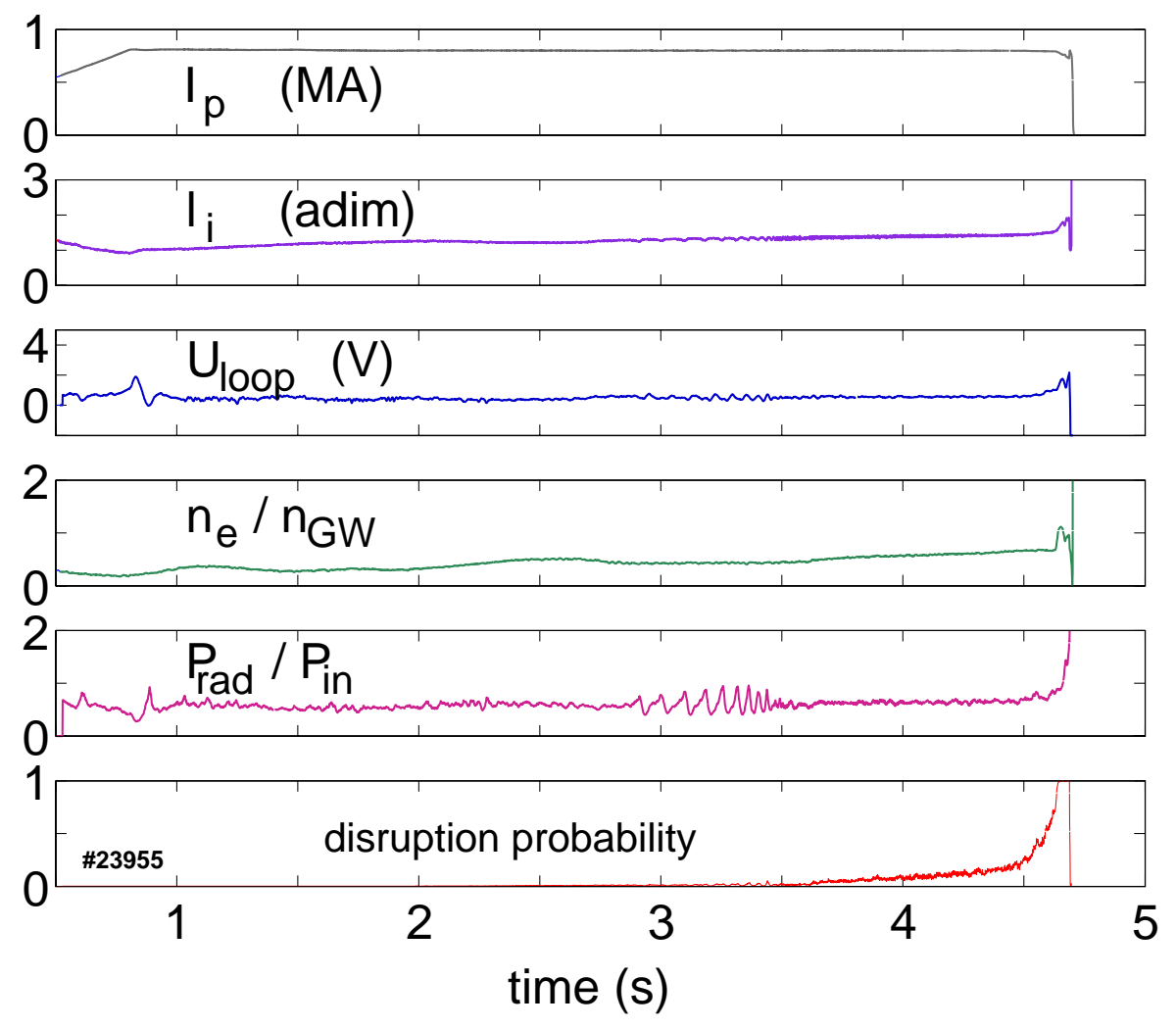

Figure 6: Probability that an edge-cooling disruption occurs and relative plasma parameter time traces: plasma current $\left(I_{p}\right)$, internal inductance $\left(l_{i}\right)$, loop voltage $\left(U_{\text {loop }}\right)$, line averaged density normalized to the Greenwald limit $\left(n_{e} / n_{G W}\right)$ and radiated power normalized to the total input power $\left(P_{\text {rad }} / P_{i n}\right)$. 

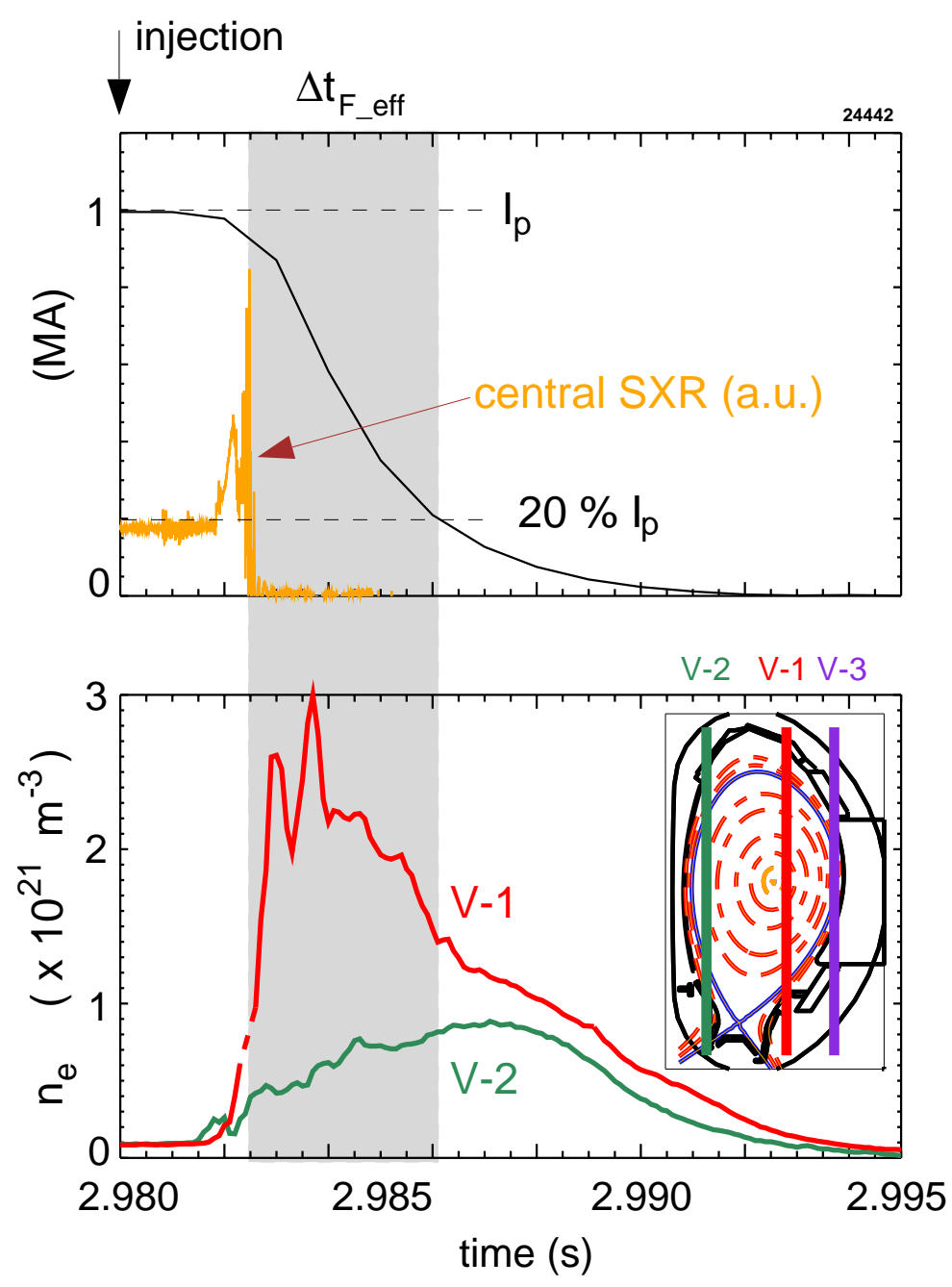

Figure 7: Time traces of plasma current $\left(I_{p}\right)$, central SXR and line averaged density, measured by the chords V-1 and V-2 of the $\mathrm{CO}_{2}$ interferometer, after injection of 3.3 bar.l of Ne. The position of the $\mathrm{CO}_{2}$ interferometer chords is shown in the inset. The fuelling efficiency is calculated in the time interval $\Delta t_{F_{e f f}}$ as explained in [31]. 

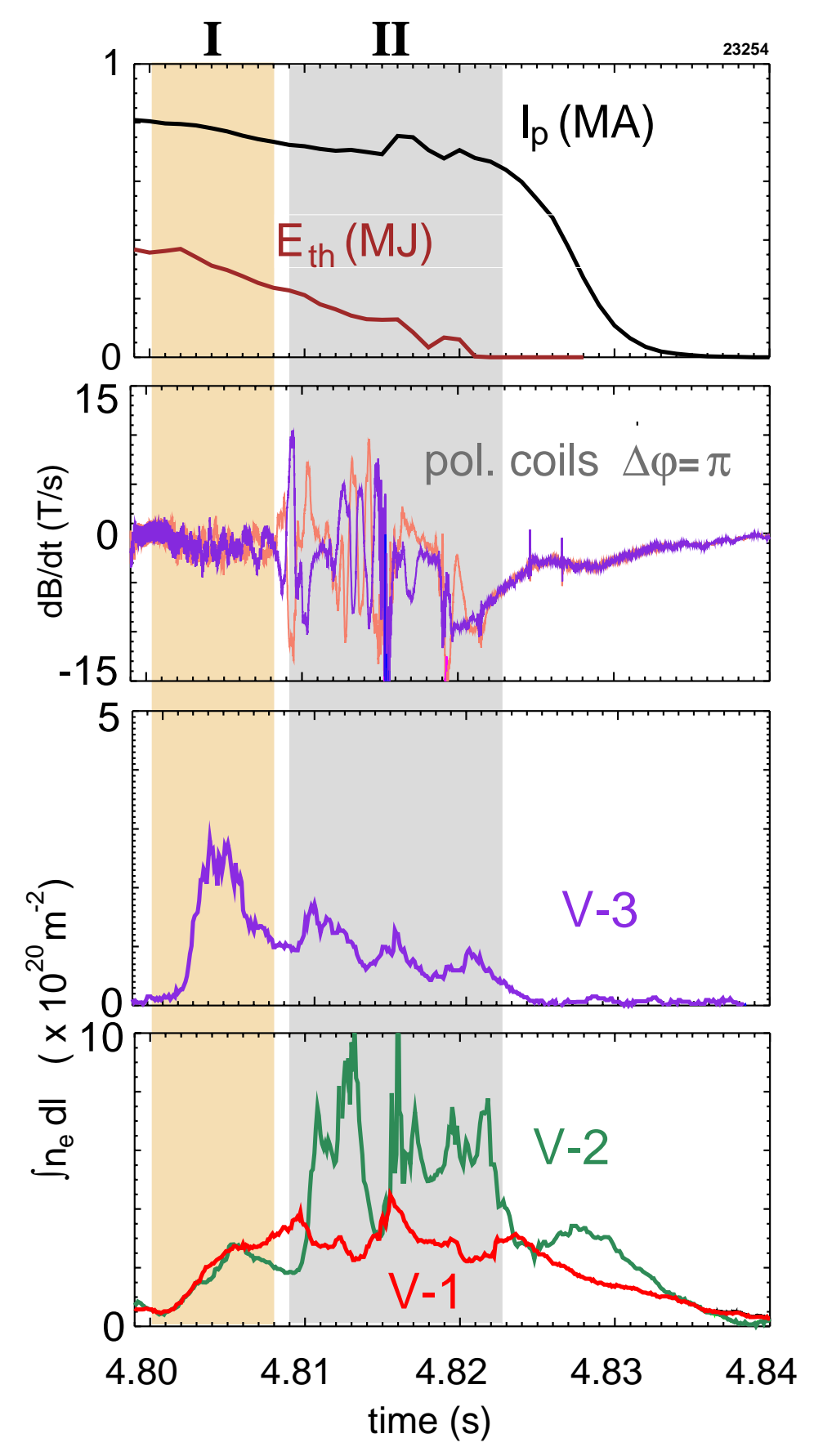

Figure 8: Time history of plasma current $\left(I_{p}\right)$, thermal energy $\left(E_{t h}\right)$, Mirnov coil signals and line integrated density $\left(\int n_{e} d l\right.$ from the $\mathrm{V}-1, \mathrm{~V}-2$ and $\mathrm{V}-3$ chords of the $\mathrm{CO}_{2}$ interferometer) after the injection of $0.2 \mathrm{bar} \cdot 1$ of He. 

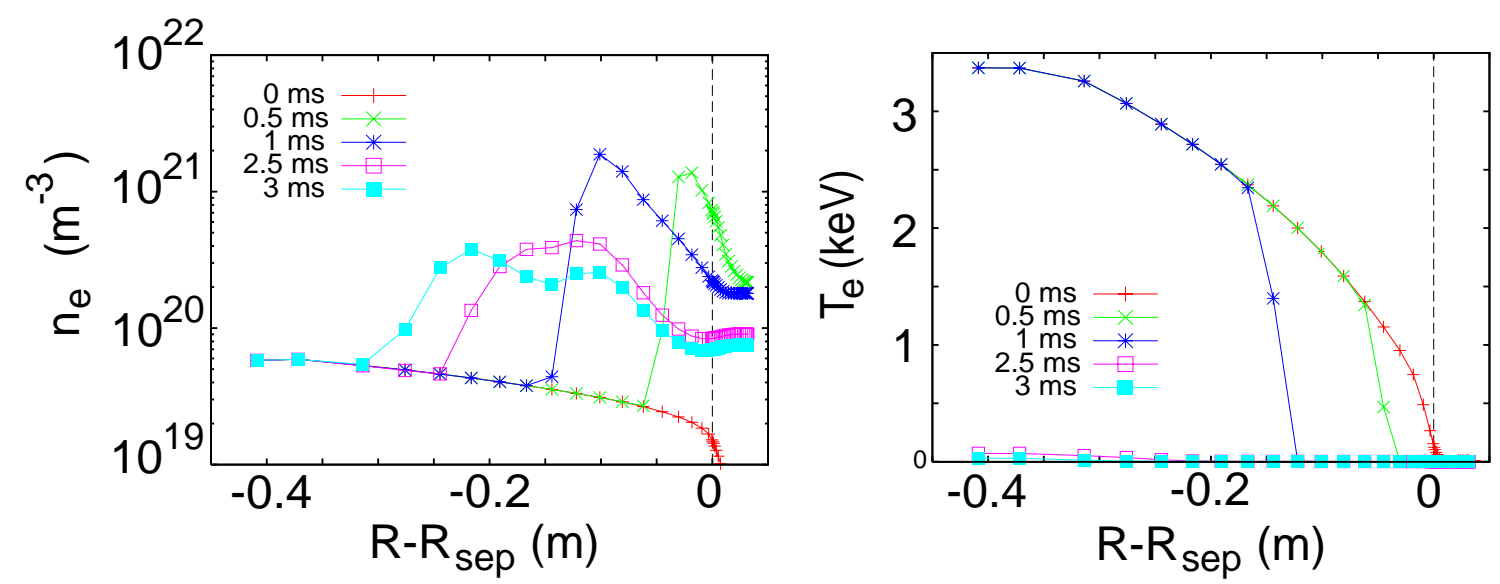

Figure 9: SOLPS modelling of the time evolution of electron density $\left(n_{e}\right)$ and temperature $\left(T_{e}\right)$ after injection of $0.4 \mathrm{mbar} \times 1$ of neon in shot 21758 [35]. 

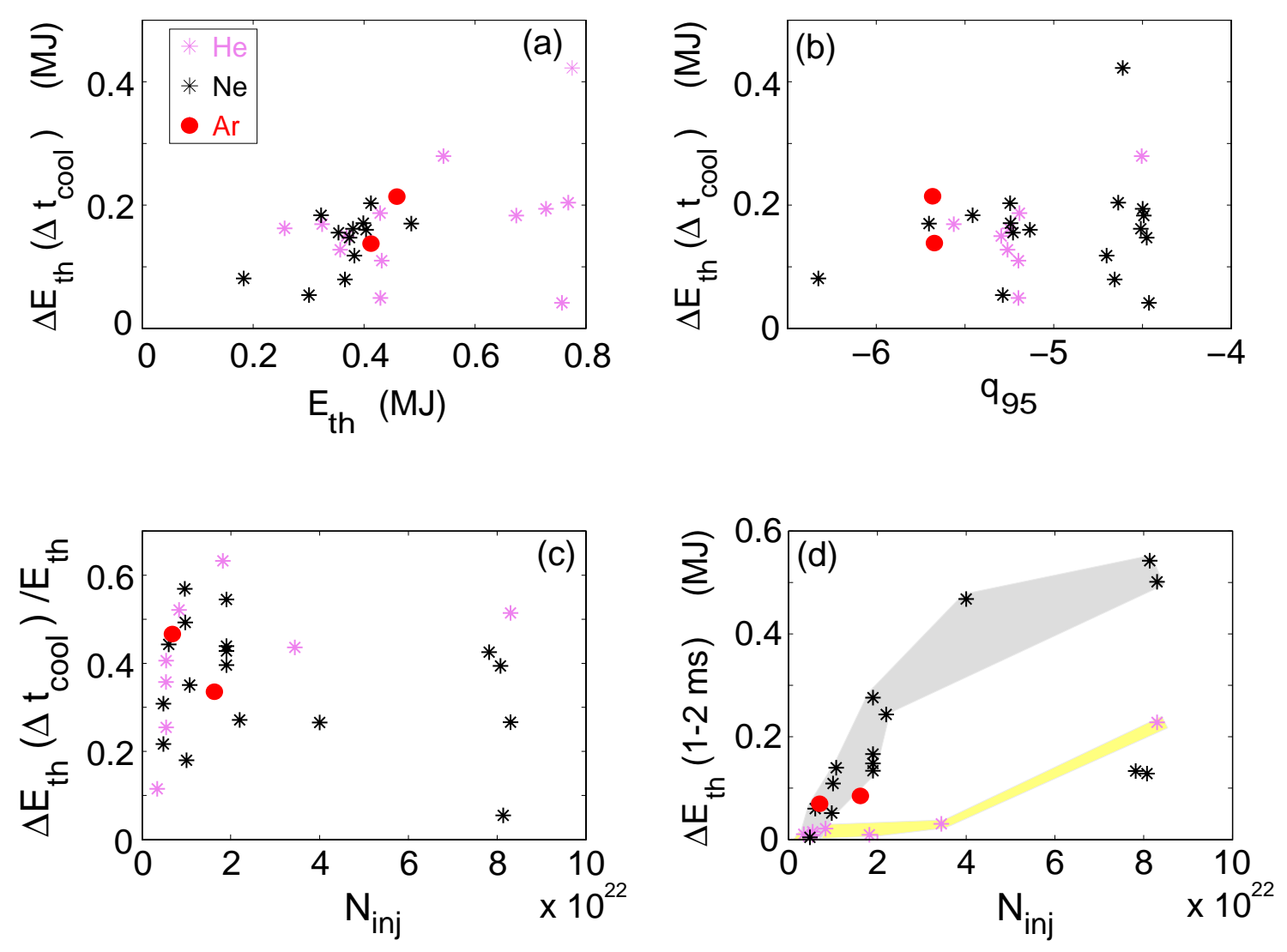

Figure 10: Amount of plasma thermal energy $\left(\Delta E_{t h}\right)$ lost mostly by radiation during the cooling quench phase $\left(\Delta t_{\text {cool }}\right)$ of a MGI disruption as function of the target thermal energy (a) $\left(E_{t h}\right)$ and (b) of $q_{95}$. (c) Fraction of the thermal energy loss and (d) energy lost in the second ms of $\Delta t_{\text {cool }}$ as function of the injected number of impurity atoms $\left(N_{i n j}\right)$. 


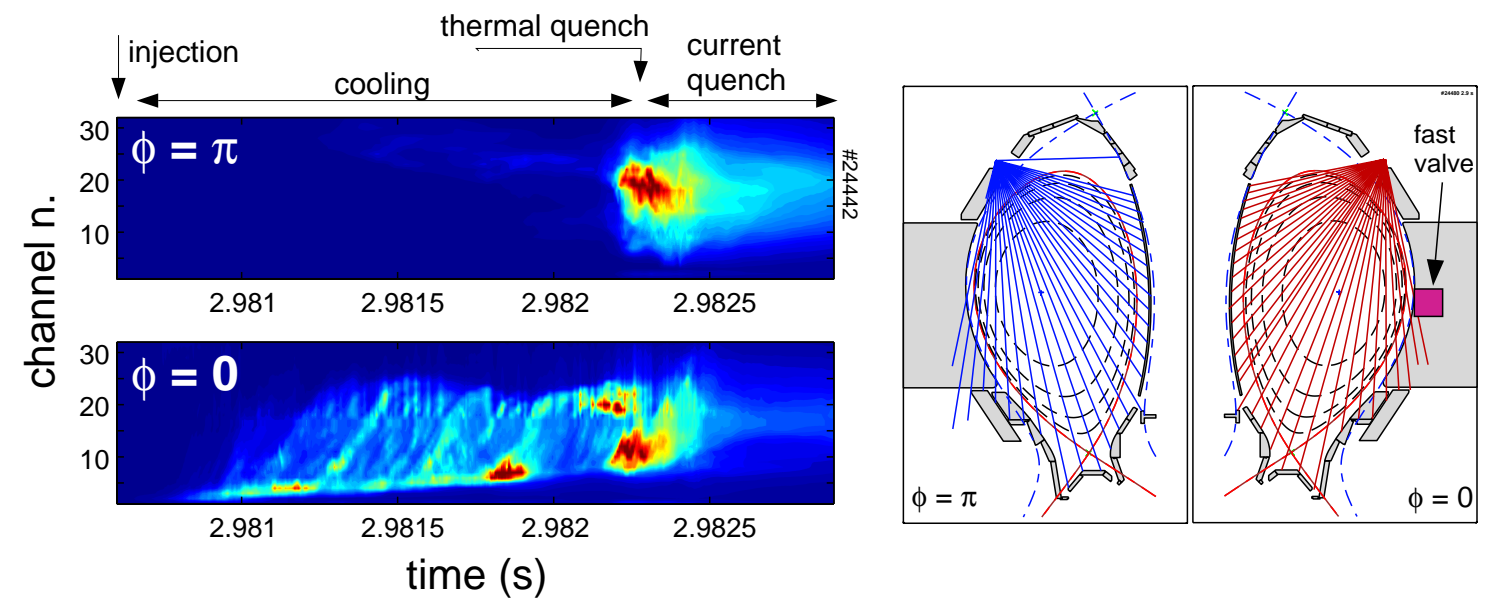

Figure 11: Left: Plasma radiation viewed by the two vertical AXUV cameras located in sector $13(\phi=0)$ and sector $5(\phi=\pi)$ after injection of $3.3 \mathrm{bar} \times 1$ of neon. Channels 1 through 32 view the plasma from the low to the high field side. Right: AXUV diagnostic geometry. 